Schweiz. Z. Path. Bakt. 1938;1:I-IV

\title{
Contents, Vol. 1, 1938
}

Schweizerische Zeitschrift

für

\section{ALLGEMEINE PATHOLOGIE UND BAKTERIOLOGIE}

Revue Suisse de Pathologie générale et de Bactériologie

Unter stäødiger Mitarbeit zahlreicher Fachgelehrter herausgegeben von

A. v. Albertini - A. Grumbach - H. Mooser

Zurich

1938

VERLAG VON S. KARGER IN BASEL

Vol. I

Alle Rechte vorbehalten

Printed in Switzerland. Druck von Friedrich Remhardt in Basel.

Inhaltsverzeichnis.

Albertiní, A. von, Studien zur Aetiologie der Arteriosklerose

Studien zur Aetiologie der Arteriosklerose . . . 163

Zur Frage der Myoblastenmyome der Zunge . . . 431 Braun, H., Ueber den Bedarf der anspruchslosen Stämme

von Diphtheriebazillen an S-, Mg- u. Fe-Verbindungen 113

Ueber das aerobe und anaerobe Wachstum der Bak-terien unter der Einwirkung von

Kaliumzyanid . . 201

2. Mitteilung. (Colibazillen, Bacillus lactis aerogenes, Typhus-, Dysenteriebazillen und Choleravibrionen) . 257

3. Mitteilung. (Diphtheriebazillen, Milzbrandbazillen

und Bacillus putrificus) 267

Dessau, F., siehe Ladewig, P.

Felix, A., Die Serumtherapie des Abdominaltyphus . . 50

Gasser, C, Ergebnisse der Pneumokokkentypisierung . . 349

Grumbach, A., u. Schnetz, A., Serologische Enterokokken-

Differenzierung

59

Hirszfeld, L., u. Kostuch, Z., Ueber das Wesen der 0-Eigen-schaft und ihre Bedeutung für die

Durchschlagskraft

der Blutgruppen

Untersuchungen über die Untergruppen und ihre

Vererbung 407

Kallós, P., Experimentelle Beiträge zur «Membranhypo-

these» der allergischen Reaktion 192

Kostuch, Z., Untersuchungen über die Blutgruppenfermente 
des Bacillus perfringens

- $\quad$ siehe Hirszfeld, L.

Kraushar-Taubenfeld, T., Untersuchungen über die Im-

munbiologie der Nekrose 335

Ladewig, P., Dessau, F., und Ottenstein, B., Beiträge zur

Kenntnis des Shopeschen Cottontail-Rabbit-Papilloms

und seines «filtrierbaren Erregers». III. Ueber den

N-Gehalt wirksamer Virusextrakte 95

Ladewig, Peter, u. Ottenstein, Berta, Beiträge zur Kenntnis

des Shopeschen Cottontail-Rabbit-Papilloms und seines

«filtrierbaren Erregers». IV. Einwirkung kolloiden

Cholesterins auf wirksame Extrakte 188

Ladewig, P., Beiträge zur Kenntnis des Shopeschen Cottontail-Rabbit-Papiiloms u. seines

«filtrierbaren Erregers». V. Ergebnisse der Herzbergschen Virusf ärbung am Material vom

Shopeschen Cottontail-Kaninchen-Papillom 240

Leitner, St. ,., Hormone und Tuberkelbazillenwachstum . 327

Levadití, C, Vaisman, A., et Manin, Y., Les doses preventives de Novarsenobenzol sont-elles les mêmes que les doses curatives du même medicament ? . . . 121

Manin, Y., siehe Levaditi, C.

Ottensooser, F., Ueber Alauntoxin. Der Mechanismus der

Entgiftung durch Alaun 315

- $\quad$ Willenegger, H., Ueber die gruppenspezifischen A-

Reaktionen von Impfstoffen, Pepton- und Pepsin-

Präparaten 421

Ottenstein, Berta, siehe Ladewig, P.

Regamey, R., et Riat, G., Recherches expérimentales sur la séro-prophylaxie et $\Gamma$ anatoxi-vaccination associées de

la diphtérie 245

Riat, G., siehe Regamey, R.

Schnetz, A., siehe Grumbach, A.

Vaisman, A., siehe Levadítí, C.

Vollmann, J., Tierexperimente mit intraossärem Arsen-,

Chrom- und Kobaltdepot $\quad 440$

Willenegger, H., siehe Ottensooser, F.

ÜBERSICHTSREFERATE - REVUES

Berblinger, W., Die Erkrankungen des Hypophysen-Zwi-

schenhirnsystems in pathogenetischer Betrachtung . 72

- Die Erkrankungen des Hypophysen-Zwischenhirn-

systems in pathogenetischer Betrachtung. Teil II . 136

Frenkel, H. S., Der jetzige Stand der Züchtung von Maul-

und Klauenseuchevirus

214

Düggeli, M., Die Mikroflora der Sauermilcharten und ihre

Verwendung 273

Levadití, C, La chimiothérapie des infections microbiennes.

Son mécanisme d'action $\quad 365$

Uehlinger, E., Experimentelle Geschwulsterzeugung mit 
radioaktiven Substanzen

444

Vorwort der Herausgeber

1

Tagesnachrichten: III. Internationaler Kongreß für Mikro-

biologie

94

Tagesnachrichten. IV. Internationaler Kongreß der ver-

gleichenden Pathologie in Rom vom 15.-20. Mai 1939

Druckfehlerberichtigung

313 